\section{DE LAS DEFINICIONES, LAS VACUNAS Y LA IDENTIFICACIÓN DEL PACIENTE SÉPTICO EN PEDIATRÍA}

Hemos leído con gran interés la Carta al Editor del doctor Montalván González publicada en un número anterior de esta revista (1), en la que, mediante un interesante razonamiento, analiza un fenómeno complejo y multifacético como el de la sepsis en el paciente pediátrico.

Algunos de los aspectos allí planteados merecen una discusión más amplia. En efecto, la sepsis severa y el choque séptico son factores importantes de riesgo de mortalidad; sin embargo, no siempre constituyen "la etapa final común de muchas enfermedades". Ambos procesos están presentes en la definición del síndrome de respuesta inflamatoria sistémica, y por muchos años han servido, en virtud de la dinámica de su desarrollo, para el estudio, la detección y la clasificación, en sus diferentes etapas, de la inflamación causada por un agente infeccioso (2). La sepsis, la sepsis severa y el choque séptico se originan en la invasión de un microorganismo y culminan en: 1) la curación por reversión del fenómeno; 2) la muerte causada por la persistencia de las alteraciones hemodinámicas y sus consecuencias; o bien 3) la disfunción orgánica múltiple, a veces con desenlace mortal $(2,3)$. Otras entidades nosológicas, como las quemaduras, la pancreatitis y la hemorragia masiva, pueden desencadenar una respuesta inflamatoria sistémica, pero no puede hablarse en estos casos de sepsis, choque séptico o sepsis grave, por no haberse originado en la acción de un agente infeccioso específico.

Factores tales como los adelantos tecnológicos de alta complejidad y la utilización de nuevos antibióticos (que son en realidad modificaciones estructurales de los ya existentes) han mejorado las tasas de mortalidad -y por ende las probabilidades de mayor supervivencia- a expensas del aumento de la morbilidad; sin embargo, la vacunación y su impacto en las cifras de morbilidad y mortalidad de los pacientes sépticos podría depender de cambios en la curva epidemiológica que durante muchos años se ha documentado, por la identificación de un microorganismo o un grupo de estos a los que se atribuye ser los más frecuentes agentes causales en diferentes cortes de tiempo $(4,5)$. Sería interesante evaluar la eficacia de la vacunación para reducir la incidencia de sepsis y la consiguiente morbilidad y mortalidad, así como investigar los efectos por grupos de edad específicos, por ejemplo el de los recién nacidos. Esta sencilla consideración se debe a que no existen aún vacunas autorizadas o usadas masivamente (con elevado impacto positivo en la salud pública) contra Streptococcus agalactiae,
Escherichia coli, Listeria monocytogenes y Candida sp, microorganismos reconocidos en los últimos años como los causantes más frecuentes de infección sistémica en ese grupo de edad (6). En los lactantes, si bien en los últimos años la vacunación masiva contra Haemophilus influenzae tipo b (Hib) y neumococo realizada en muchos países ha demostrado su eficacia, otros microorganismos han tomado la delantera como agentes causales de sepsis, entre ellos las infecciones causadas por Staphylococcus aureus meticilino resistente (7), enterobacterias o incluso serotipos de neumococos no incluidos en la vacuna neumocócica heptavalente.

El problema principal radica en una identificación certera del paciente séptico, que al padecer un proceso dinámico de características y desarrollo muy variables, puede en cuestión de minutos, horas o días evolucionar fácilmente de una sepsis grave a un choque séptico, y en ocasiones a disfunción orgánica múltiple, con un cuadro clínico que plantea diversos diagnósticos diferenciales (8).

La discusión lleva ya muchos años, y aún no se ha llegado a consensos y propuestas de identificación que satisfagan la necesidad de detectar oportuna y correctamente la sepsis en un niño. Hoy en día, la introducción de nuevas técnicas de biología molecular para la identificación del ADN de microorganismos está revolucionando la medicina, pues el tiempo para la obtención de resultados se ha reducido prácticamente a horas, es decir que ha aumentado de manera notable la velocidad de identificación de los microorganismos aislados (9).

Se ha empleado la reacción en cadena de la polimerasa (RCP) para la identificación de secuencias conservadas de los genes bacterianos del ácido ribonucleico ribosomal (rRNA) 16S, 23S, y las regiones intermedias entre ambos. Además, la RCP se ha empleado para el diagnóstico de bacterias especie-específicas, y también se ha adaptado para la búsqueda de un gran número de bacterias (RCP multiplex) que suelen detectarse con muy baja frecuencia, o cuando se presume conocido el agente causal (10-12). No obstante, el empleo de muestras sanguíneas para el diagnóstico de sepsis presenta algunos inconvenientes, ya que se ha observado la existencia de inhibidores de la RCP, además de las dificultades inherentes a disponer de técnicas capaces de detectar una amplia variedad de bacterias como agentes etiológicos $(13,14)$. Así, la prueba de SeptiFast ${ }^{\circledR}$ permite identificar 25 microorganismos, que representan $90 \%$ de las especies aisladas en pacientes sépticos (11). Se la ha utilizado para mejorar, con resultados muy alentadores, los porcentajes de aislamiento de bacterias causantes de enfermedades tales como endocarditis infecciosa, sepsis en pacientes neutropénicos con neoplasias hematológicas, bacteriemias o fungemias (12-14). 
El uso de la biología molecular para identificar microorganismos en sangre requiere menor cantidad de muestra y los resultados se obtienen en menor tiempo, en comparación con el hemocultivo; todo ello favorece la pronta iniciación de un tratamiento antimicrobiano dirigido. Debido al impacto de la sepsis como causa de morbilidad y mortalidad en las unidades de cuidados intensivos y al alto costo de la estancia hospitalaria, la utilización habitual de estas técnicas podría ser la mejor opción a largo plazo, aunque inicialmente la disponibilidad y el precio no estén al alcance de muchos.

Jesús Reyna-Figueroa Centro Nacional para la Salud de la Infancia y la Adolescencia (CeNSIA)

Coordinación de Información e Investigación Francisco de P. Miranda 177, $2^{\circ}$ piso, Colonia Merced Gómez, Delegación Álvaro Obregón, México, D.F.

Teléfono: 56802347

Correo electrónico: jesusreynaf@prodigy.net.mx Vesta Richardson-López Collada Directora General del CeNSIA Patricia Vidal-Vázquez Subdirectora Técnica de Salud de la Infancia del CeNSIA

\section{REFERENCIAS}

1. Montalván GLG. Sepsis severa y shock séptico en el niño. Aún no todo está dicho. Rev Panam Salud Publica/Pan Am J Public Health. 2009;26(6):562-4.

2. Goldstein B, Giroir B, Randolph A, et al. International pediatric sepsis consensus conference: Definitions for sepsis and organ dysfunction in pediatrics. Pediatr Crit Care Med. 2005; $6(1): 2-8$.
3. Reyna-Figueroa J, Ortiz Ibarra FJ, Navarro Godínez S, Pérez Antonio B. Recién nacidos pretérmino con sepsis nosocomial: comparación de dos consensos y una escala clínica, utilizados en la identificación de sepsis mediante un estudio de evaluación de pruebas diagnósticas. Rev Enfer Infec Pediatr. 2008; 22(85):18-23.

4. Stoll B, Hansen N, Fanaroff A, Wright L, Carlo W, Ehrenkranz $\mathrm{MD}$, et al. Changes in pathogens causing early-onset sepsis in very-low-birth-weight infants. N Engl J Med. 2002;347(4): 240-7.

5. Saltigeral Simental P, Valenzuela Flores AA, Avendaño Barroeta E, Plascencia Inclán S, Martínez Nogués D. Agentes causales de sepsis neonatal temprana y tardía: una revisión de diez años en el "Hospital Infantil Privado". Rev Enfer Infec Pediatr. 2007;20(80):99-105.

6. Klein J. Bacterial sepsis and meningitis. En: Remington JS, Klein JO, eds. Infectious diseases of the fetus and newborn infant. Philadelphia: W.B. Saunders; 2001. Pp. 943-98.

7. Mandell G, Bennett J, Dolin R. Enfermedades infecciosas. Principios y práctica. $6^{\mathrm{a}}$ ed. Barcelona: Elsevier; 2006. Pp. 2352-9.

8. Reyna-Figueroa J, Toala Yuri E, Ortiz Ibarra IFJ, Rodríguez Ramírez E, Limón Rojas AE. Disparidad en los criterios para incluir pacientes con sepsis neonatal en estudios médicos científicos. ¿Nadamos en un mar sin límites? An Pediatr (Barc). 2006;65(6):536-40.

9. Tenover F, Arbeit R, Goering R, Mickelsen P, Murray B, Persing D, et al. Interpreting chromosomal DNA restriction patterns produced by pulsed-field gel electrophoresis: Criteria for bacterial strain typing. J Clin Microbiol 1995;33(9):2233-9.

10. Yi-dong W, Li-hua C, Xiu-jing W, Shi-qiang S, Jin-tu L, Li-zhong, et al. Gram-specific probes based real time PCR for detection and discrimination of bacterial neonatal sepsis. J Clin Microbiol. 2008;46(8):2613-9.

11. Lau A, Chen S, Sleiman S, Sorrell T. Current status and future perspectives on molecular and serological methods in diagnostic mycology. Future Microbiol. 2009;4(9):1185-222.

12. Mancini N, Clerici D, Diotti R, Perotti M, Ghidoli N, De Marco D, et al. Molecular diagnosis of sepsis in neutropenic patients with haematological malignancies. J Med Microbiol. 57(5):601-4.

13. Steinmann J, Buer J, Rath P-M, Paul A, Saner F. Invasive aspergillosis in two liver transplant recipients: diagnosis by SeptiFast. Transpl Infect Dis. 2009:11(2):175-8.

14. Varani S, Stanzani M, Nardi L, Paolucci M, Vianelli N, Baccarani M, et al. Comparison of real-time PCR and blood culture for the diagnosis of bloodstream infections in onco-haematologic patients: microbiological and clinical assessment: Clinical Microbiology \& Infection. 2007;13(suppl 1):S393. 